\title{
Author Correction: Indirect tail states formation by thermal-induced polar fluctuations in halide perovskites
}

Bo Wu (1) ${ }^{1,2}$, Haifeng Yuan (D) ${ }^{3}$, Qiang Xu (D) ${ }^{2}$, Julian A. Steele ${ }^{4}$, David Giovanni ${ }^{2}$, Pascal Puech ${ }^{5}$, Jianhui Fu², Yan Fong Ng6,7, Nur Fadilah Jamaludin 6,7, Ankur Solanki², Subodh Mhaisalkar @ 6,7, Nripan Mathews ${ }^{6,7}$, Maarten B.J. Roeffaers ${ }^{4}$, Michael Grätzel ${ }^{7,8}$, Johan Hofkens (iD ${ }^{3} \&$ Tze Chien Sum (D) ${ }^{2}$

Correction to: Nature Communications; https://doi.org/10.1038/s41467-019-08326-7; published online 29 January 2019.

The original version of this article incorrectly listed the present address of Bo Wu as 'Present address: Institute of Electronic Paper Displays, South China Academy of Advanced Optoelectronics, South China Normal University, Guangzhou, Guangdong Province 510006, China'. This is the author's primary affiliation. This has been corrected in both the PDF and HTML versions of the article.

Published online: 05 March 2019

(c) Open Access This article is licensed under a Creative Commons Attribution 4.0 International License, which permits use, sharing, adaptation, distribution and reproduction in any medium or format, as long as you give appropriate credit to the original author(s) and the source, provide a link to the Creative Commons license, and indicate if changes were made. The images or other third party material in this article are included in the article's Creative Commons license, unless indicated otherwise in a credit line to the material. If material is not included in the article's Creative Commons license and your intended use is not permitted by statutory regulation or exceeds the permitted use, you will need to obtain permission directly from the copyright holder. To view a copy of this license, visit http://creativecommons.org/licenses/by/4.0/.

(C) The Author(s) 2019

\footnotetext{
${ }^{1}$ Institute of Electronic Paper Displays, South China Academy of Advanced Optoelectronics, South China Normal University, Guangzhou, Guangdong Province 510006, China. ${ }^{2}$ Division of Physics and Applied Physics, School of Physical and Mathematical Sciences, Nanyang Technological University, 21 Nanyang Link, Singapore 637371, Singapore. ${ }^{3}$ Department of Chemistry, KU Leuven, Celestijnenlaan 200F, B-3001 Leuven, Belgium. ${ }^{4}$ Centre for Surface Chemistry and Catalysis, KU Leuven, Celestijnenlaan 200F, 3001 Leuven, Belgium. ${ }^{5}$ CEMES/CNRS, University of Toulouse, 31055 Toulouse, France. ${ }^{6}$ School of Materials Science and Engineering, Nanyang Technological University, 50 Nanyang Avenue, Singapore 639798, Singapore. ${ }^{7}$ Energy Research Institute @NTU (ERI@N), Research Techno Plaza, X-Frontier Block Level 5, 50 Nanyang Drive, Singapore 637553, Singapore. ${ }^{8}$ Laboratory of Photonics and Interfaces, Department of Chemistry and Chemical Engineering, Swiss Federal Institute of Technology, Station 6, 1015 Lausanne, Switzerland. These authors contributed equally: Bo Wu, Haifeng Yuan. Correspondence and requests for materials should be addressed to J.H. (email: johan.hofkens@chem.kuleuven.be) or to T.C.S. (email: Tzechien@ntu.edu.sg)
} 